\title{
PD-L1 in small bowel adenocarcinoma is associated with etiology and tumor-infiltrating lymphocytes, in addition to microsatellite instability
}

\author{
Paolo Giuffrida ${ }^{1}$ et al.
}

Received: 20 November 2019 / Revised: 25 January 2020 / Accepted: 27 January 2020 / Published online: 17 February 2020

(c) The Author(s), under exclusive licence to United States \& Canadian Academy of Pathology 2020

\begin{abstract}
Small bowel adenocarcinomas (SBAs) are often associated with poor prognosis and have limited therapeutic options. Programmed cell death protein-1 (PD-1)/programmed cell death ligand 1 (PD-L1) pathway blockade is an effective treatment in many microsatellite instability-high (MSI-H) solid tumors. We aimed at investigating PD-L1 and PD-1 expression in nonhereditary, non-ampullary SBAs, associated with celiac disease $(\mathrm{CeD})$, Crohn's disease $(\mathrm{CrD})$, or sporadic, recruited through the Small Bowel Cancer Italian Consortium. We assessed PD-L1 and PD-1 by immunohistochemistry in a series of 121 surgically resected SBAs, including 34 CeD-SBAs, 49 CrD-SBAs, and 38 sporadic SBAs. PD-L1 and PD-1 expression was correlated with several clinico-pathological features, such as the etiology, microsatellite instability status, and tumorinfiltrating lymphocyte (TIL) density. The prevalence of PD-L1 positivity according to combined positive score (CPS) was $26 \%$ in the whole cohort of SBAs, with significantly $(p=0.001)$ higher percentage $(35 \%)$ in both CeD-SBAs and CrD-SBAs in comparison with sporadic SBAs (5\%). CPS $\geq 1$ SBAs were significantly $(p=0.013)$ more frequent in MSI-H cases $(41 \%)$ than in non-MSI-H ones (18\%); however, 15 CPS $\geq 1$ microsatellite stable SBAs were also identified. CPS $\geq 1$ SBAs showed higher TIL and PD- $1^{+}$immune cell density, more frequently medullary histotype, as well as a better outcome in comparison with CPS $<1$ cases. This study demonstrates an increased proportion of PD-L1 ${ }^{+}$cases in both CeD-SBAs and CrD-SBAs in comparison with sporadic SBAs. In addition, the identification of a subset of PD-L1 ${ }^{+}$microsatellite stable SBAs supports the need to ascertain additional biomarkers of response to immune checkpoint inhibitors along with MSI-H.
\end{abstract}

\section{Introduction}

Small bowel adenocarcinomas (SBAs) are rare neoplasms often associated with dismal prognosis owing to lack of symptoms until advanced stages [1,2]. Previous studies demonstrated that the predisposing immune-mediated intestinal disorder, namely celiac disease (CeD) or Crohn's disease (CrD), was a stage-independent prognostic

These authors contributed equally: Paolo Giuffrida and Giovanni Arpa

These authors jointly supervised this work: Alessandro Vanoli and Antonio Di Sabatino

Supplementary information The online version of this article (https:// doi.org/10.1038/s41379-020-0497-0) contains supplementary material, which is available to authorized users.

Antonio Di Sabatino

a.disabatino@smatteo.pv.it

Extended author information available on the last page of the article factor, as patients with CeD-associated SBA (CeD-SBA) showed a more favorable outcome in comparison with those affected by CrD-associated SBA (CrD-SBA) [3, 4]. Regardless of the etiology, i.e., $\mathrm{CeD}$ or $\mathrm{CrD}$, further prognostic factors for SBA encompass stage, glandular versus non-glandular histotype and density of tumor-infiltrating $\mathrm{T}$ lymphocytes (TILs) [3, 4]. Unlike CrD-SBAs and sporadic SBAs, CeD-SBAs are frequently characterized by microsatellite instability-high (MSI-H) and increased TIL density, thus probably leading to increased immune surveillance against cancer and a better clinical outcome in CeD-SBAs $[2,3,5]$.

Programmed cell death protein-1 (PD-1) and programmed cell death ligand 1 (PD-L1) are pivotal immune checkpoints of the immune system response. They provide a negative feedback that inhibits $\mathrm{T}$ helper 1 cytotoxic immune responses, causing T-cell exhaustion or tolerance [6]. Recently, it has been demonstrated that blockade of PD1 and PD-L1 is an effective treatment in a number of MSI-H solid tumors, including gastric and colorectal carcinomas 
[7, 8], as microsatellite instability (MSI) status has been demonstrated to predict clinical response to immune checkpoint inhibitors $[8,9]$. Recent studies found a strong positive correlation between PD-L1 expression and MSI-H in SBAs [10-12]. Although MSI-H is one of the main determinants of tumor mutation load, leading to PD-L1 expression in gastrointestinal cancers, other drivers might be implicated [13]. Indeed, a recent study [14] showed that PD-L1 is also present in a subset of microsatellite stable endometrial carcinomas with high TILs. Moreover, TIL density, Epstein-Barr virus (EBV) infection, and CDX2 negativity have been shown to be associated with PD-L1 positivity in gastrointestinal carcinomas [15-17]. Assessment of the tumor immune microenvironment, in particular PD-L1 expression, TIL density and tumor mutation load, is therefore under investigation, in order to find possible predictors of response to immune checkpoint blockades [18].

On this basis, the aim of this study was to investigate the expression of PD-L1 and PD-1 in a relatively wide and well-characterized cohort of non-hereditary SBAs, associated with $\mathrm{CeD}$ or $\mathrm{CrD}$ or sporadic, recruited through the Small Bowel Cancer Italian Consortium. PD-L1 and PD-1 expression was correlated with several clinico-pathological features, such as the predisposing immune-mediated intestinal disorder, the MSI or EBV status, the intestinal phenotype markers CDX2 and liver fatty acid-binding protein (L-FABP), and cancer-specific survival.

\section{Materials and methods}

\section{Patients}

This is a retrospective, longitudinal study, which involved 21 tertiary referral Italian Celiac and/or IBD Centers participating in the Small Bowel Cancer Italian Consortium.

$\mathrm{CeD}$ diagnosis was based on serum IgA anti-endomysial and anti-tissue transglutaminase antibody positivity associated with typical duodenal histopathological lesions [19]. $\mathrm{CrD}$ diagnosis was ascertained according to internationally agreed criteria [20], and the site and extent of the disease were confirmed by endoscopy, histology, and imaging. A group of patients with sporadic SBA, i.e., without a concomitant intestinal immune-mediated disorder, were included as a control group. In sporadic SBAs, CeD was excluded (serum IgA anti-endomysial and anti-tissue transglutaminase antibody negativity, along with normal serum total $\operatorname{IgA}$ ), whereas $\mathrm{CrD}$ was ruled out by the absence of classic clinical and biochemical features. Re-examination of the sporadic surgical specimens further confirmed the lack of histological lesions indicative of either $\mathrm{CeD}$ or $\mathrm{CrD}$. The main exclusion criteria for all SBA groups were Lynch syndrome, Peutz-Jeghers syndrome, familial adenomatous polyposis, and juvenile polyposis. This study was approved by the Ethics Committee of the Fondazione IRCCS San Matteo Hospital in Pavia (protocol number 20140003980). Data are reported according to the STROBE recommendations.

\section{Histology, immunohistochemistry, and MSI analysis}

Tissue samples were fixed in $4 \%$ formaldehyde and processed in paraffin wax. Four $\mu \mathrm{m}$-thick sections were stained with haematoxylin-eosin for morphological evaluation. All cases were investigated for histotype [4], and all the parameters required to fulfill the criteria of the AJCC staging system [21]. For immunohistochemistry, $4 \mu \mathrm{m}$-thick sections were stained on a Dako Omnis platform with the following antibodies: CD3 (polyclonal, Dako, Carpinteria, CA), CD8 (polyclonal, Dako), MLH1 (monoclonal, clone ES05, Dako), MSH2 (monoclonal, clone FE11, Dako), MSH6 (monoclonal, clone EP49, Dako), PMS2 (monoclonal, clone EP51, Dako), PD-L1 (monoclonal, clone 22C3, Dako), PD-1 (monoclonal, clone NAT, Dako), CDX2 (monoclonal, clone DAK-CDX2, Dako), and LFABP (monoclonal, clone EPR20464, Dako). Immunoreactions were developed using $0.03 \% 3,3^{\prime}$ diaminobenzidine tetrahydrochloride and sections were then counterstained with Harris' haematoxylin. TILs were stained using CD3 and CD8 antibodies and counted in 10 consecutive high-power fields (HPFs), as previously described [3]. A tumor was classified as having "high TIL density" when the mean number of TILs/HPF was $>15$ for CD3 or $>9.5$ for CD8 [22]. Immunostaining of DNA mismatch repair proteins MLH1, MSH2, MSH6, and PMS2 in tumor cells was evaluated as proficient (retained expression) or deficient (absent expression); only tumors showing absence of nuclear staining of all neoplastic cells in the presence of an internal positive control (intra-tumor stromal and inflammatory cells or non-tumor mucosa) were considered deficient [3]. In parallel, MSI molecular analysis was performed as previously reported [3].

PD-L1 membranous expression was evaluated using the combined positive score (CPS) measuring both tumoral cells and peritumoral/intratumoral immune cells, the mononuclear immune cell density score (MIDS) measuring peritumoral/ intratumoral immune cells only and the tumor proportion score (TPS) measuring tumoral cells only, as previously described [23]. In particular, CPS was calculated as the ratio of the number of PD-L1-stained cells (tumor cells and immune cells) to the total number of viable tumor cells, multiplied by 100 . Tumors were considered negative if CPS $<1$, positive if CPS $\geq 1$. TPS was the ratio of the number of PD-L1-stained tumor cells divided by the total number of viable tumor cells, multiplied by 100 . Tumors were regarded as negative if TPS $<1$, positive if TPS $\geq 1$. MIDS was calculated as the ratio of the number of PD-L1-stained immune 
cells to the total number of viable tumor cells, multiplied by 100; the result was then scored in a scale from 0 to 4 . MIDS 0 was defined as absent PD-L1 staining, whereas MIDS 1, MIDS 2, MIDS 3, and MIDS 4 corresponded to PD-L1 ${ }^{+}$ immune cells per 100 viable tumor cells $<1, \geq 1$ but $<10$, $\geq$ 10 but $<100, \geq 100$, respectively. MIDS scores 2,3 , and 4 were regarded as positive, whereas scores 0 and 1 as negative. PD- $1^{+}$immune cells were counted separately in intratumoral and peritumoral areas in 10 consecutive HPFs and the mean number of PD- ${ }^{+}$cells per HPF was recorded for each case. PD- $1^{+}$cells situated inside the tumor were considered as intratumoral, whereas PD- $1^{+}$cells located in the areas adjacent to the tumor invasive front as peritumoral. In addition, the total number of PD- ${ }^{+}$cells per HPF, corresponding to the sum of intratumoral and peritumoral $\mathrm{PD}-1^{+}$cell counts was given. L-FABP or CDX2 staining was considered positive in cases with $>10 \%$ moderate-to-intense staining in tumor cells [24]. A central pathology review of each case was performed. All haematoxylin-eosin and immunohistochemistry-stained tumors were assessed by two independent researchers (GA and AVa). Cases with evaluation discrepancies between the two investigators had their slides re-analyzed together with an expert gastrointestinal pathologist (ES), until a consensus was reached.

\section{EBV encoded RNAs in situ hybridization}

The formalin-fixed, paraffin-embedded tissue sections were analyzed for the expression of EBV encoded small RNAs, markers of latent phase EBV infection, as previously described [25].

\section{Definition of Teng tumor microenvironment immune types}

Tumor microenvironment immune types were defined based on $\mathrm{CD}^{+}$TIL density (low versus high) and PD-L1 expression, evaluated with the CPS. Tumors were classified in four different Teng types, i.e., type I (high TIL density, CPS $\geq 1$ ), II (low TIL density, CPS $<1$ ), III (low TIL density, CPS $\geq 1$ ), IV (high TIL density, CPS $<1$ ) [26].

\section{Statistical analysis}

Stata 15 (StataCorp, College Station, TX, USA) was used to perform all analyses. A two-sided $p$ value was considered statistically significant. For post hoc comparisons between etiologic groups, the significance was set at 0.017 (Bonferroni correction). Continuous data were reported as median and 25-75th percentiles, categorical variables are reported as counts and percent; they were compared between etiologic groups using the Kruskal-Wallis test or the Fisher exact test, respectively. The Spearman $R$ and
95\% confidence intervals (CI) were computed to measure the correlation between continuous variables. For the purpose of the analysis continuous variables were dichotomized at the median value. Median follow-up (25-75th) was computed with the reverse Kaplan-Meier method. Follow-up was computed from diagnosis of cancer to death or last available follow-up for censored patients. Cumulative survival curves were plotted according to the Kaplan-Meier method and compared with the logrank test. The strength of the association between series of candidate risk factors and cancer-specific mortality was assessed using Cox regression; hazard ratios and 95\% CI were derived from the models. Mortality rates per 100 person-year and 95\% CIs were reported. Owing to the limited number of events, only bivariable models were fitted to adjust, in turn, for etiologic group and stage.

\section{Results}

\section{Patient demographics and clinico-pathologic features}

This retrospective study included a cohort of 121 patients with pathologically confirmed primary non-hereditary, nonampullary SBA, who had surgical resection and complete survival data. Demographic and clinico-pathologic data of all patients evaluated are reported in Table 1 . We recruited 34 patients with CeD-SBA, 49 with CrD-SBA, and 38 with sporadic SBA, a fraction of them entered previous studies from the Small Bowel Cancer Italian Consortium $[3,4,25,27,28]$. Median age at the time of SBA diagnosis among celiac (median 53.5 years) and $\mathrm{CrD}$ patients (median 58 years) was significantly $(p<0.001)$ lower than that of sporadic cases (median 69 years), and median duration of inflammatory disorder at cancer diagnosis was significantly ( $p=0.016)$ lower in CeD-SBA (median 23.5 months) in comparison with CrD-SBA (median 156 months). A higher rate of male gender was observed in CrD-SBA (73\%) and sporadic SBA (63\%) in comparison with the CeD-SBA group (47\%). In agreement with what is already known [2], the commonest small bowel location was the ileum for CrDSBA (94\%), whereas it was the jejunum in both CeD-SBA (70\%) and sporadic SBA (63\%). No significant difference was found among the three groups regarding tumor stage at diagnosis. The majority of stage III and IV patients received systemic chemotherapy with platinum-based and 5fluorouracil regimens after surgical intervention.

Histologically, most SBAs showed glandular differentiation in all etiologic groups; however, medullary and diffuse/poorly cohesive cancers were more common in $\mathrm{CeD}$ $(17 \%)$ and $\mathrm{CrD}$ patients (20\%), respectively. CeD-SBAs exhibited a significantly $(p<0.001)$ greater number of TILs 
Table 1 Demographic and clinico-pathologic features of all 121 SBA patients.

\begin{tabular}{|c|c|c|c|c|c|}
\hline & CeD-SBA & CrD-SBA & $\begin{array}{l}\text { Sporadic } \\
\text { SBA }\end{array}$ & $\begin{array}{l}\text { Overall } \\
p \text { value }\end{array}$ & $\begin{array}{l}\text { Post hoc comparison } \\
p \text { value }\end{array}$ \\
\hline Number & 34 & 49 & 38 & & \\
\hline $\begin{array}{l}\text { Age at SBA diagnosis } \\
\text { Median (25-75th IQR), yrs }\end{array}$ & $\begin{array}{l}53.5 \\
(42.7-66)\end{array}$ & $\begin{array}{l}58 \\
(51-67.5)\end{array}$ & $\begin{array}{l}69 \\
(62-77)\end{array}$ & $<0.001$ & $\begin{array}{l}\text { CeD vs CrD: } 0.129 \\
\text { CeD vs sporadic: }<0.001 \\
\text { CrD vs sporadic: }<0.001\end{array}$ \\
\hline $\begin{array}{l}\text { Duration of inflammatory disorder at SBA diagnosis } \\
\text { Median (25-75th IQR), mo }\end{array}$ & $\begin{array}{l}23.5 \\
(12-110.25)\end{array}$ & $\begin{array}{l}156 \\
(6-288)\end{array}$ & NA & 0.016 & \\
\hline \multicolumn{6}{|l|}{ Sex, N (\%) } \\
\hline $\begin{array}{l}\text { Female } \\
\text { Male }\end{array}$ & $\begin{array}{l}18(53) \\
16(47)\end{array}$ & $\begin{array}{l}13(27) \\
36(73)\end{array}$ & $\begin{array}{l}14(37) \\
24(63)\end{array}$ & 0.049 & $\begin{array}{l}\text { CeD vs CrD: } 0.014 \\
\text { CeD vs sporadic: } 0.169 \\
\text { CrD vs sporadic: } 0.302\end{array}$ \\
\hline \multicolumn{6}{|l|}{ Site, $\mathrm{N}(\%) *$} \\
\hline $\begin{array}{l}\text { Duodenum } \\
\text { Jejunum } \\
\text { Ileum }\end{array}$ & $\begin{array}{l}7(21) \\
23(70) \\
3(9)\end{array}$ & $\begin{array}{l}1(2) \\
2(4) \\
46(94)\end{array}$ & $\begin{array}{l}3(8) \\
24(63) \\
11(29)\end{array}$ & $<0.001$ & $\begin{array}{l}\mathrm{CeD} \text { vs } \mathrm{CrD}:<0.001 \\
\mathrm{CeD} \text { vs sporadic: } 0.053 \\
\text { CrD vs sporadic: }<0.001\end{array}$ \\
\hline \multicolumn{6}{|l|}{ Stage, $\mathrm{N}(\%)^{* *}$} \\
\hline $\begin{array}{l}\text { I } \\
\text { II } \\
\text { III } \\
\text { IV }\end{array}$ & $\begin{array}{l}3(9) \\
19(60) \\
8(25) \\
2(6)\end{array}$ & $\begin{array}{l}6(12) \\
19(39) \\
18(37) \\
6(12)\end{array}$ & $\begin{array}{l}2(5) \\
17(46) \\
15(41) \\
3(8)\end{array}$ & 0.550 & \\
\hline \multicolumn{6}{|l|}{ Histotype, $\mathrm{N}(\%)$} \\
\hline $\begin{array}{l}\text { Glandular } \\
\text { Medullary } \\
\text { Diffuse } \\
\text { Mixed } \\
\text { Solid }\end{array}$ & $\begin{array}{l}19(56) \\
6(17) \\
2(6) \\
4(12) \\
3(9)\end{array}$ & $\begin{array}{l}24(50) \\
2(4) \\
10(20) \\
12(24) \\
1(2)\end{array}$ & $\begin{array}{l}22(58) \\
1(3) \\
2(5) \\
10(26) \\
3(8)\end{array}$ & 0.032 & $\begin{array}{l}\text { CeD vs CrD: } 0.032 \\
\text { CeD vs sporadic: } 0.187 \\
\text { CrD vs sporadic: } 0.228\end{array}$ \\
\hline $\begin{array}{l}\mathrm{CD}^{+} \text {TILs/HPF } \\
\text { Median (25-75th IQR) }\end{array}$ & $\begin{array}{l}25.1 \\
(12.3-75.4)\end{array}$ & $\begin{array}{l}7.1 \\
(2-20.6)\end{array}$ & $\begin{array}{l}7.1 \\
(2.2-20.9)\end{array}$ & $<0.001$ & $\begin{array}{l}\text { CeD vs } \mathrm{CrD}:<0.001 \\
\text { CeD vs sporadic: }<0.001 \\
\text { CrD vs sporadic: } 0.962\end{array}$ \\
\hline \multicolumn{6}{|l|}{ MSI status, N (\%) } \\
\hline $\begin{array}{l}\text { Non-MSI } \\
\text { MSI-H }\end{array}$ & $\begin{array}{l}12(35) \\
22(65)\end{array}$ & $\begin{array}{l}40(82) \\
9(18)\end{array}$ & $\begin{array}{l}30(79) \\
8(21)\end{array}$ & $<0.001$ & $\begin{array}{l}\text { CeD vs } \mathrm{CrD}:<0.001 \\
\text { CeD vs sporadic: }<0.001 \\
\text { CrD vs sporadic: } 0.754\end{array}$ \\
\hline \multicolumn{6}{|l|}{ CDX2 expression, $\mathrm{N}(\%) * * *$} \\
\hline $\begin{array}{l}\text { Negative } \\
\text { Positive }\end{array}$ & $\begin{array}{l}5(15) \\
28(85)\end{array}$ & $\begin{array}{l}22(46) \\
26(54)\end{array}$ & $\begin{array}{l}11(29) \\
27(71)\end{array}$ & 0.012 & $\begin{array}{l}\text { CeD vs CrD: } 0.003 \\
\text { CeD vs sporadic: } 0.165 \\
\text { CrD vs sporadic: } 0.109\end{array}$ \\
\hline \multicolumn{6}{|l|}{$L-F A B P$ expression, $\mathrm{N}(\%) * * * *$} \\
\hline $\begin{array}{l}\text { Negative } \\
\text { Positive }\end{array}$ & $\begin{array}{l}30(88) \\
4(12)\end{array}$ & $\begin{array}{l}39(81) \\
9(19)\end{array}$ & $\begin{array}{l}17(45) \\
21(55)\end{array}$ & $<0.001$ & $\begin{array}{l}\text { CeD vs CrD: } 0.393 \\
\text { CeD vs sporadic: }<0.001 \\
\text { CrD vs sporadic: }<0.001\end{array}$ \\
\hline
\end{tabular}

$C e D-S B A$ celiac disease-associated small bowel adenocarcinoma, $C r D-S B A$ Crohn's disease-associated small bowel adenocarcinoma, $H P F$ highpower field, $I Q R$ interquartile range, $L-F A B P$ liver fatty acid-binding protein, mo month, $M S I$ microsatellite instability, $M S I-H$ microsatellite instability-high, $N A$ not applicable, $S B A$ small bowel adenocarcinoma, $T I L$ tumor-infiltrating lymphocyte, $y r$ year.

*In one CeD-SBA, the precise tumor site within small bowel was unknown.

**In two CeD-SBAs and in one sporadic SBA, the precise stage was unknown.

***In one CeD-SBA and in one CrD-SBA, no section for CDX2 immunohistochemistry was available.

*****In one CrD-SBA, no section for L-FABP immunohistochemistry was available.

(median 25.1 TILs/HPF) in comparison with the remaining etiologic groups (median 7.1 TILs/HPF for both CrD-SBAs and sporadic SBAs). MSI-H was found in 39 cases (32.2\%), including 37 cases with loss of MLH1/PMS2 expression and two SBAs, both associated with CrD, with isolated loss of MSH6. No discordance between immunohistochemistry for mismatch repair proteins and MSI molecular analysis was observed in any case. MSI-H rate was significantly $(p<0.001)$ higher in CeD-SBAs $(65 \%)$ than in both CrD-SBAs (18\%) and sporadic SBAs (21\%). Regarding markers of intestinal differentiation, $\mathrm{CDX} 2$ loss was significantly $(p=0.012)$ more common in CrD-SBAs (46\%) in comparison with CeD-SBAs $(15 \%)$, whereas the lack of L-FABP expression was significantly $(p<0.001)$ more frequent in both CeD-SBAs (88\%) and CrD-SBAs $(81 \%)$ in comparison with sporadic SBAs $(45 \%)$.

\section{Immunohistochemical expression of PD-L1 and association with clinico-pathologic features}

PD-L1 staining was observed in immune cells and to a variable extent in tumor cells (Fig. 1). PD-L1 expression 
Fig. 1 PD-L1 and PD-1 expression in small bowel adenocarcinomas (SBAs). A glandular celiac diseaseassociated SBA showing a strong and diffuse PD-L1 membranous staining on both tumoral and immune cells a (PD-L1 staining; original magnification $\times 200$ ) and a high number of PD- $1^{+}$intratumoral lymphocytes b (PD-1 staining; original magnification $\times 400$ ) . c An $\mathrm{EBV}^{+}$lymphoepitheliomalike Crohn's disease-associated SBA with a diffuse PD-L1 membranous expression by tumor cells (PD-L1 staining; original magnification $\times 200$ ). d A glandular Crohn's diseaseassociated SBA with a low intratumoral infiltration of PD- $1^{+}$cells. (PD-1 staining; original magnification $\times 400$ ). e A glandular sporadic SBA showing a faint membranous and cytoplasmatic PD-L1 staining on immune cells, localized either in the tumoral stroma or in the lumen of neoplastic glands. (PDL1 staining; original magnification $\times 200)$. f The same sporadic case exhibiting a low number of intratumoral PD$1^{+}$lymphocytes (PD-1 staining; original magnification $\times 400$ ).

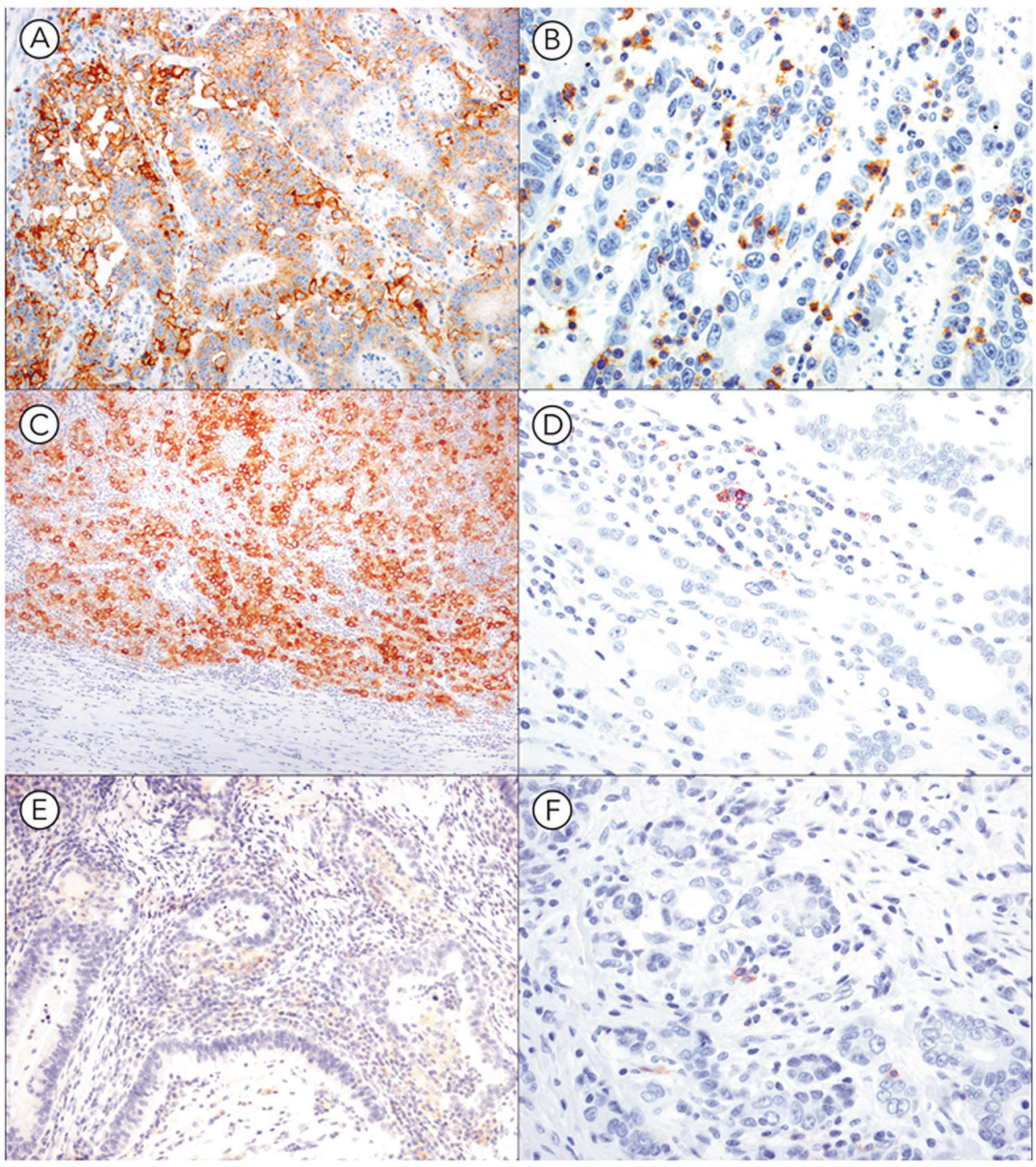

according to CPS and MIDS was positively associated with male sex, whereas no significant association was found between PD-L1 expression and age at SBA diagnosis, small bowel site and tumor stage at diagnosis (Table 2, Supplementary Table 1).

Per CPS cutoff of 1 or more (CPS $\geq 1$ ), the prevalence of PD-L1 expression in non-hereditary SBAs as a whole was $26 \%$, with significantly $(p=0.001)$ higher percentage in both CeD-SBA (35\%) and CrD-SBA (35\%) than in sporadic SBAs (5\%). Among CPS $\geq 1$ SBAs, $65 \%$ (20 out of 31 cases, including $8 \mathrm{CeD}-\mathrm{SBAs}, 10 \mathrm{CrD}-\mathrm{SBAs}$ and 2 sporadic SBAs), $13 \%$ (4 cases, including one CeD-SBA and $3 \mathrm{CrD}-$ SBAs) and 23\% (7 cases, including 3 CeD-SBAs and 4 CrD-SBAs) showed a $1 \leq \mathrm{CPS}<10, \quad 10 \leq \mathrm{CPS}<50$, and CPS $\geq 50$, respectively. Per TPS $\geq 1$, the prevalence of PDL1 expression was $8 \%$, with significantly $(p=0.035)$ higher percentage in both CeD-SBA (15\%) and CrD-SBA (10\%) than in sporadic SBA $(0 \%)$. Per MIDS $>1$, the prevalence of PD-L1 expression was $22 \%$, with significantly $(p=$
0.005) higher percentage in both CeD-SBAs (29\%) and CrD-SBAs (31\%) than in sporadic SBAs (5\%). No case with MIDS 4 was identified in our whole cohort.

Patients with MSI-H SBAs had a significantly $(p=$ $0.013)$ higher prevalence of CPS $\geq 1(41 \%)$ than those with non-MSI SBAs (18\%). Patients with MSI-H SBAs had a significantly $(p=0.002)$ higher prevalence of TPS $>1$ $(20 \%)$ than those with microsatellite stable SBAs (2\%). No significant difference in terms of PD-L1 positivity according to MIDS was observed between MSI-H and microsatellite stable SBAs. In addition, PD-L1 positivity was associated with several features known to be more often associated with MSI-H phenotype, including high $\mathrm{CD}^{+}$ and $\mathrm{CD}^{+}$TIL density and medullary histotype.

SBAs with CPS $\geq 1$ exhibited L-FABP negativity significantly more frequently $(p=0.036)$ in comparison with those with $\mathrm{CPS}<1$. A similar trend, despite not being significant, was shown in SBAs showing TPS $\geq 1$ or MIDS $>1$. L-FABP negativity was more frequent in MSI-H SBAs 
Table 2 Association of PD-L1 expression according to CPS with clinico-pathologic features.

\begin{tabular}{|c|c|c|c|}
\hline & $\begin{array}{l}\text { PD-L1 }{ }^{-} \\
(\mathrm{CPS}<1)\end{array}$ & $\begin{array}{l}\mathrm{PD}-\mathrm{L} 1^{+} \\
(\mathrm{CPS} \geq 1)\end{array}$ & $p$ value \\
\hline Number, $N(\%)$ & $90(74)$ & $31(26)$ & \\
\hline $\begin{array}{l}\text { Age at SBA diagnosis } \\
\text { Median (25-75th IQR) }\end{array}$ & $62(52-71)$ & $56(52-67)$ & 0.421 \\
\hline \multicolumn{4}{|l|}{$\operatorname{Sex}, \mathrm{N}(\%)$} \\
\hline $\begin{array}{l}\text { Female } \\
\text { Male }\end{array}$ & $\begin{array}{l}38(86) \\
51(67)\end{array}$ & $\begin{array}{l}6(14) \\
25(33)\end{array}$ & 0.029 \\
\hline Etiologic group, $\mathrm{N}(\%)$ & & & 0.001 \\
\hline $\begin{array}{l}\text { CeD-SBA } \\
\text { CrD-SBA } \\
\text { Sporadic SBA }\end{array}$ & $\begin{array}{l}22(65) \\
32(65) \\
36(95)\end{array}$ & $\begin{array}{l}12(35) \\
17(35) \\
2(5)\end{array}$ & \\
\hline Site, $\mathrm{N}(\%)$ & & & 0.172 \\
\hline $\begin{array}{l}\text { Duodenum } \\
\text { Jejunum } \\
\text { Ileum }\end{array}$ & $\begin{array}{l}8(73) \\
41(84) \\
41(68)\end{array}$ & $\begin{array}{l}3(27) \\
8(16) \\
19(32)\end{array}$ & \\
\hline Stage, N (\%) & & & 0.212 \\
\hline $\begin{array}{l}\text { I } \\
\text { II } \\
\text { III } \\
\text { IV }\end{array}$ & $\begin{array}{l}8(73) \\
39(71) \\
30(73) \\
11(100)\end{array}$ & $\begin{array}{l}3(27) \\
16(29) \\
11(27) \\
0(0)\end{array}$ & \\
\hline Histotype, $\mathrm{N}(\%)$ & & & 0.011 \\
\hline $\begin{array}{l}\text { Glandular } \\
\text { Medullary } \\
\text { Diffuse } \\
\text { Mixed } \\
\text { Solid }\end{array}$ & $\begin{array}{l}49(75) \\
2(22) \\
12(86) \\
21(81) \\
6(86)\end{array}$ & $\begin{array}{l}16(25) \\
7(78) \\
2(14) \\
5(19) \\
1(14)\end{array}$ & \\
\hline $\begin{array}{l}\mathrm{PD}-1^{+} \text {intratumoural cells/HPF } \\
\text { Median }(25-75 \text { th IQR) }\end{array}$ & $\begin{array}{l}1.1 \\
(0.1-5.5)\end{array}$ & $\begin{array}{l}7.2 \\
(2.8-28.5)\end{array}$ & $<0.001$ \\
\hline $\begin{array}{l}\text { PD- } 1^{+} \text {peritumoural cells/HPF } \\
\text { Median }(25-75 \text { th IQR) }\end{array}$ & $\begin{array}{l}1.9 \\
(0.4-7.8)\end{array}$ & $\begin{array}{l}13.8 \\
(5.3-29.3)\end{array}$ & $<0.001$ \\
\hline $\begin{array}{l}\mathrm{PD}-1^{+} \text {total cells/HPF } \\
\text { Median }(25-75 \text { th IQR) }\end{array}$ & $\begin{array}{l}2.8 \\
(1.0-16.2)\end{array}$ & $\begin{array}{l}23.8 \\
(8.0-64.3)\end{array}$ & $<0.001$ \\
\hline $\begin{array}{l}\mathrm{CD}^{+} \text {TILs/HPF } \\
\text { Median }(25-75 \text { th IQR) }\end{array}$ & $\begin{array}{l}7.0 \\
(2.1-24.2)\end{array}$ & $\begin{array}{l}21.3 \\
(15.1-75.2)\end{array}$ & $<0.001$ \\
\hline $\begin{array}{l}\mathrm{CD}^{+} \text {TILs/HPF } \\
\text { Median (25-75th IQR) }\end{array}$ & $\begin{array}{l}5.2 \\
(1.0-19.3)\end{array}$ & $\begin{array}{l}52.5 \\
(10.7-93.0)\end{array}$ & 0.008 \\
\hline MSI status, $\mathrm{N}(\%)$ & & & 0.013 \\
\hline $\begin{array}{l}\text { Non-MSI } \\
\text { MSI-H }\end{array}$ & $\begin{array}{l}67(82) \\
23(59)\end{array}$ & $\begin{array}{l}15(18) \\
16(41)\end{array}$ & \\
\hline CDX2 expression, $\mathrm{N}(\%)$ & & & 1.000 \\
\hline $\begin{array}{l}\text { Negative } \\
\text { Positive }\end{array}$ & $\begin{array}{l}29(76) \\
60(74)\end{array}$ & $\begin{array}{l}9(24) \\
21(26)\end{array}$ & \\
\hline$L-F A B P$ expression, $\mathrm{N}(\%)$ & & & 0.036 \\
\hline $\begin{array}{l}\text { Negative } \\
\text { Positive }\end{array}$ & $\begin{array}{l}59(69) \\
30(88)\end{array}$ & $\begin{array}{l}27(31) \\
4(12)\end{array}$ & \\
\hline
\end{tabular}

$C e D-S B A$ celiac disease-associated small bowel adenocarcinoma, $C P S$ combined positive score, $C r D-S B A$ Crohn's disease-associated small bowel adenocarcinoma, $H P F$ high-power field, IQR interquartile range, $L-F A B P$ liver fatty acid-binding protein, $M S I$ microsatellite instability, $M S I-H$ microsatellite instability-high, $P D-1$ programmed cell death protein-1, $P D-L 1$ programmed cell death ligand 1, SBA small bowel adenocarcinoma, TIL tumor-infiltrating lymphocyte.
(82\%) than in microsatellite stable cases $(66 \%)$, although this did not reach statistical significance $(p=0.065)$. No significant difference in PD-L1 expression was found in CDX2-positive and CDX2-negative SBAs.

\section{Immunohistochemical expression of PD-1}

Data on PD-1 were obtained in 118 patients, 34 with CeDSBAs, 49 with CrD-SBAs, and 35 with sporadic SBAs. PD1 positivity was observed in both intratumoral and peritumoral immune cells. PD- $1^{+}$intratumoral immune cells were significantly higher in CeD-SBAs (median 5.4/HPF, 25-75th IQR: $1.3-16.4)$ than in CrD-SBAs $(1.2,25-75$ th: $0.5-3.7 ; p=0.002)$ and sporadic SBAs $(0.9,25-75$ th: $0-7.7 ; p=0.001)$. Likewise, PD- $1^{+}$peritumoral immune cells were significantly higher in CeD-SBAs $(8.25,25-75$ th: 3.6-20.2) than in CrD-SBAs (3.6, 25-75th: 1.0-9.0; $p=$ $0.007)$ and sporadic SBAs (1.9, 25-75th: $0.1-9.3 ; p=$ $0.001)$. With regards to PD- $1^{+}$immune cells, no significant difference was observed between CrD-SBAs and sporadic SBAs. PD- $1^{+}$immune cells were significantly $(p<0.001)$ higher in CPS $\geq 1$ cases than in CPS $<1$ cases (Table 2), as well as in MIDS $>1$ cases in comparison with MIDS $\leq 1$ cases. Only $\mathrm{PD}-1^{+}$peritumoral immune cells were significantly ( $p=0.041$ ) higher in TPS $\geq 1$ cases in comparison with TPS $<1$ cases (Supplementary Table 1). PD- $1^{+}$intratumoral, peritumoral, and total cells density resulted associated with $\mathrm{CD}^{+}$TIL density $(R=0.46,95 \%$ CI $0.3-0.59$, $p<0.001 ; R=0.37,95 \%$ CI $0.2-0.52, p<0.001 ; R=0.42$, $95 \%$ CI $0.26-0.56, p<0.001$, respectively).

\section{Teng tumor microenvironment immune types}

Among 121 non-hereditary SBAs, Teng type I, II, III, and IV encompassed 24 (20\%), 62 (51\%), 7 (6\%), and 28 (23\%) cases, respectively. Teng types according to MSI status and etiologic group are summarized in Table 3. Distribution of Teng types was significantly $(p<0.001)$ different among MSI-H and microsatellite stable SBAs. In particular, MSI-H SBAs included an increased number of Teng types with high TIL density, i.e., type I (31\%) and IV (46\%). Most microsatellite stable SBAs (69\%) had no PD-L1 expression and low TIL density, i.e., type II. However, we also found 12 microsatellite stable SBAs with high TIL density and positive PD-L1 expression (type I), including three CeDSBAs, eight CrD-SBAs, and one sporadic SBA, as well as three type III microsatellite stable SBAs, all of them associated with $\mathrm{CrD}$. In addition, five MSI-H SBAs with low TIL density and negative PD-L1 expression (i.e., type II) were found. Likewise, distribution of Teng types was significantly $(p<0.001)$ different among CeD-SBAs, CrDSBAs, and sporadic SBAs. We observed an increased 
Table 3 Distribution of SBAs according to Teng type classification (in four tumor microenvironment immune types), MSI status, and etiologic group.

\begin{tabular}{|c|c|c|c|c|c|}
\hline & \multicolumn{4}{|c|}{ Teng type classification } & \multirow[t]{2}{*}{$p$ value } \\
\hline & $\begin{array}{l}\text { Type I } \\
\text { (PD-L1 }^{+} \\
\text {/high TIL } \\
\text { density) }\end{array}$ & $\begin{array}{l}\text { Type II } \\
\text { (PD-L1 }^{-} \\
\text {/low TIL } \\
\text { density) }\end{array}$ & $\begin{array}{l}\text { Type III } \\
\text { (PD-L1 }^{+} \\
\text {/low TIL } \\
\text { density) }\end{array}$ & $\begin{array}{l}\text { Type IV } \\
\text { (PD-L1 }^{-} \\
\text {/high TIL } \\
\text { density) }\end{array}$ & \\
\hline Total, $N(\%)$ & $24(20)$ & $62(51)$ & $7(6)$ & $28(23)$ & \\
\hline \multicolumn{6}{|c|}{ MSI status, $\mathrm{N}(\%)$} \\
\hline $\begin{array}{l}\text { Non-MSI } \\
\text { MSI-H }\end{array}$ & $\begin{array}{l}12(15) \\
12(31)\end{array}$ & $\begin{array}{l}57(69) \\
5(13)\end{array}$ & $\begin{array}{l}3(4) \\
4(10)\end{array}$ & $\begin{array}{l}10(12) \\
18(46)\end{array}$ & $<0.001$ \\
\hline \multicolumn{6}{|c|}{ Etiologic group, N (\%) } \\
\hline $\begin{array}{l}\text { CeD-SBA } \\
\text { CrD-SBA } \\
\text { Sporadic SBA }\end{array}$ & $\begin{array}{l}11(32) \\
11(23) \\
2(5)\end{array}$ & $\begin{array}{l}9(27) \\
27(55) \\
26(69)\end{array}$ & $\begin{array}{l}1(3) \\
6(12) \\
0(0)\end{array}$ & $\begin{array}{l}13(38) \\
5(10) \\
10(26)\end{array}$ & $<0.001$ \\
\hline
\end{tabular}

$C e D-S B A$ celiac disease-associated small bowel adenocarcinoma, $C r D-S B A$ Crohn's disease-associated small bowel adenocarcinoma, $M S I$ microsatellite instability, $M S I-H$ microsatellite instability-high, $P D-L 1$ programmed cell death ligand 1, SBA small bowel adenocarcinoma, TIL tumor-infiltrating lymphocyte.

For Teng type classification, PD-L1 expression was evaluated with the combined positive score.

proportion of type I in patients with CeD-SBAs (32\%) and CrD-SBAs (23\%) in comparison with sporadic SBAs (5\%).

\section{EBV $^{+}$SBAs}

Of the 118 tumors investigated for EBV encoded RNAs, i.e., 34 CeD-SBAs, 49 CrD-SBA, and 35 sporadic SBA, only two were $\mathrm{EBV}^{+}$, both $\mathrm{CrD}-\mathrm{SBAs}$, one showing a lymphoepithelioma-like and the other a glandular histology, as described in a previous study [25]. The lymphoepitheliomalike carcinoma was strongly $\mathrm{PD}-\mathrm{L1}^{+}$with a $\mathrm{CPS} \geq 50$, whereas the glandular CrD-SBA was negative for PD-L1.

\section{Survival analysis}

Three patients died peri-operatively, whereas the remaining 118 patients were followed for a median of 68 (25-75th: $35-117)$ months and their cancer-specific survival data are reported in Supplementary Table 2. Univariate survival analysis identified the following parameters as significant related to a better post-operative cancer-specific survival: etiologic group-in particular CeD-SBA-, female sex, tumor site- jejunum being better-, pathological stage I and II, MSI-H, high $\mathrm{CD}^{+}$TIL density, medullary and glandular histotype; positivity for CDX2 expression, high intratumoral PD- $1^{+}$cell density, peritumoral PD- $1^{+}$cell density, and total PD- $1^{+}$cell density (Fig. 2 and Supplementary Figure 1). PD$\mathrm{L}^{+}$cases according to a CPS showed better outcome in comparison with PD-L1 ${ }^{-}$cases $(p=0.046$, Cox analysis and Fig. 3a). Teng type classification proved to be a significant prognostic parameter ( $p<0.001$, Fig. $3 b)$; in particular, type I and IV tumors showed a significantly better cancer-specific survival in comparison to type II. However, PD-L1 positivity lost its prognostic value when combined with TIL density in Teng type classification. TPS, MIDS and L-FABP positivity were not found to be significant predictors of cancer-specific survival (Supplementary Figure 2).

Bivariable survival analysis inclusive of stage confirmed the significant prognostic value of the following parameters: etiologic group $(p=0.017)$, sex $(p=0.007)$, histotype $(p=0.01)$, MSI status $(p=0.002), \mathrm{CD}^{+}$TIL density $(p=$ $0.001)$, CPS $(p=0.022)$ and Teng type classification $(p=$ $0.015)$. At bivariable analysis inclusive of etiologic group, the following factors retained prognostic significance: stage $(p<0.001)$, sex $(p=0.008)$, histotype $(p<0.001)$, MSI status $(p=0.016), \mathrm{CD}^{+}$TIL density $(p=0.002)$, and Teng type classification $(p=0.018)$. PD $1^{+}$immune cell density lost its prognostic value in a bivariable model adjusted for $\mathrm{CD}^{+}$TIL density or MSI status.

\section{Discussion}

We herein systematically assessed the tumor immune microenvironment in a large series of SBAs associated with $\mathrm{CeD}$ or $\mathrm{CrD}$. In agreement with previous findings [10, 11], we observed that PD-L1 was less frequently expressed in cancer cells than in immune cells in SBAs. Furthermore, in agreement with Thota et al. [10], we demonstrated that PD$\mathrm{L}^{+}$SBAs have a higher number of intratumoral, peritumoral, and total PD- $1^{+}$immune cells. We found that the prevalence of PD-L1 expression in non-hereditary SBAs as a whole was $26 \%, 8 \%$, and $22 \%$ according to CPS, TPS, and MIDS, respectively. This is the first study to demonstrate an increased proportion of $\mathrm{PD}-\mathrm{Ll}^{+}$cases in both $\mathrm{CeD}-\mathrm{SBAs}$ and CrD-SBAs compared with sporadic SBAs, regardless of the score used, i.e., CPS, TPS, or MIDS. In addition, we demonstrated a significant increase in PD- $1^{+}$immune cells in CeD-SBAs compared with CrD-SBAs and sporadic SBAs. Indeed, a recent transcriptomic profiling study identified a predominant MSI-immune subtype of CeD-SBA characterized by high PD-1 activation [28]. On this basis, response to PD-L1/PD-1 pathway blockade treatments might be more frequently expected in CeD-SBAs and-likely to a lesser extent-in CrD-SBAs in comparison with sporadic cases. Several clinical trials with immune checkpoint inhibitors for the treatment of SBA patients are ongoing [2]. In particular, in the ZEBRA trial the activity of pembrolizumab was assessed in 40 patients with previously treated advanced SBA [29]. Preliminary results of the ZEBRA trial showed that pembrolizumab did not significantly increase the overall response rate, although efficacy was observed in MSI-H patients and, interestingly, also in some microsatellite stable 
A Kaplan-Meier survival estimate, by etiologic group

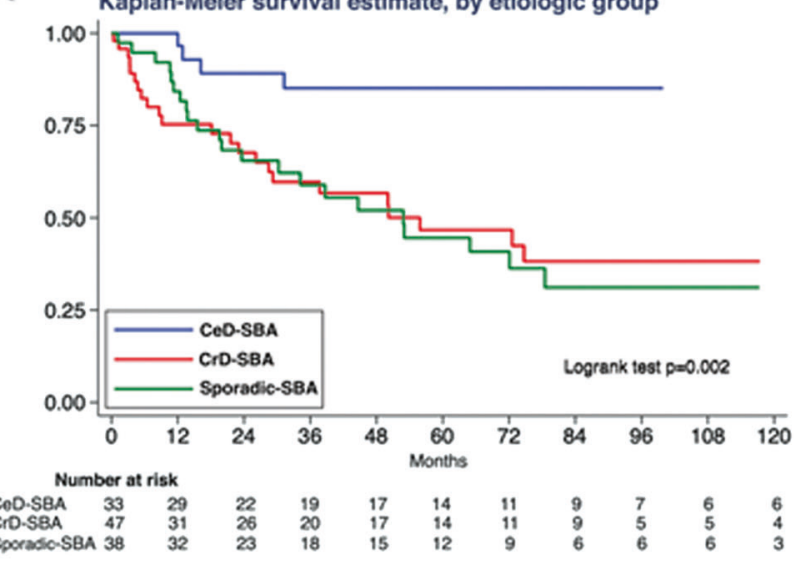

C

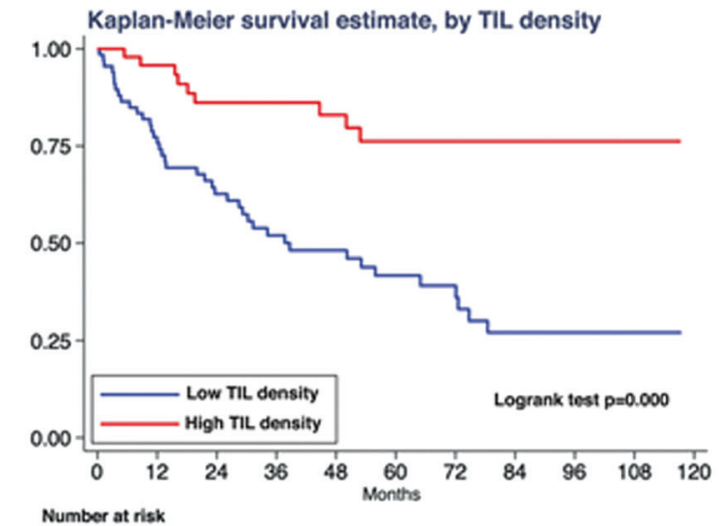

\begin{tabular}{lccccccccccc}
\multicolumn{1}{c}{ Number at risk } & & & & & & & \\
Low TIL donsity & 67 & 50 & 37 & 27 & 23 & 19 & 13 & 9 & 8 & 8 & 6 \\
High TIL density & 51 & 42 & 34 & 30 & 26 & 21 & 18 & 15 & 10 & 9 & 7
\end{tabular}

E

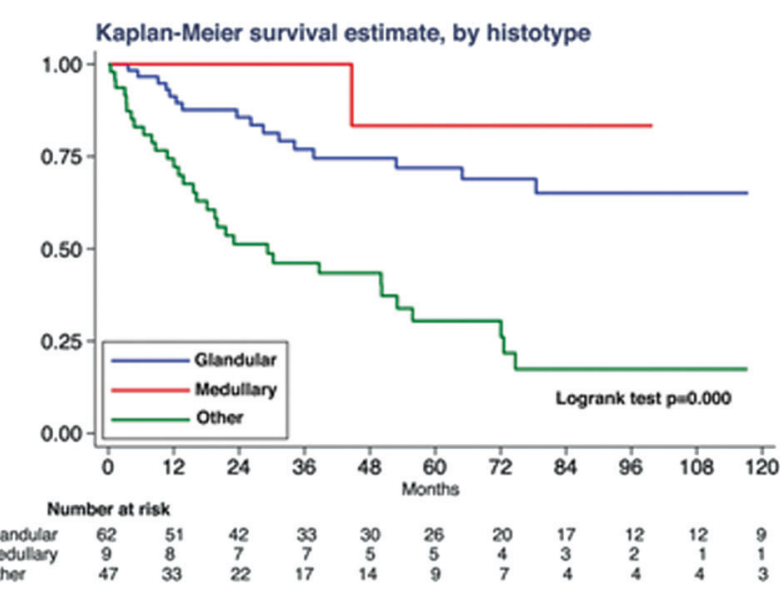

Fig. 2 Kaplan-Meier cancer-specific survival estimates for small bowel adenocarcinoma (SBA) patients. Survival estimates by etiologic group a, stage $\mathbf{b}$, tumor-infiltrating lymphocyte (TIL) density c, microsatellite instability (MSI) status d, histotype e, and CDX2

patients. No data regarding the tumor immune microenvironment are still available from the study [29]. Further trials are mandatory in these settings of patients, including those with CeD-SBA and CrD-SBA.

The higher expression of PD-L1 found in CeD-SBAs and CrD-SBAs compared with sporadic SBAs and the increase in

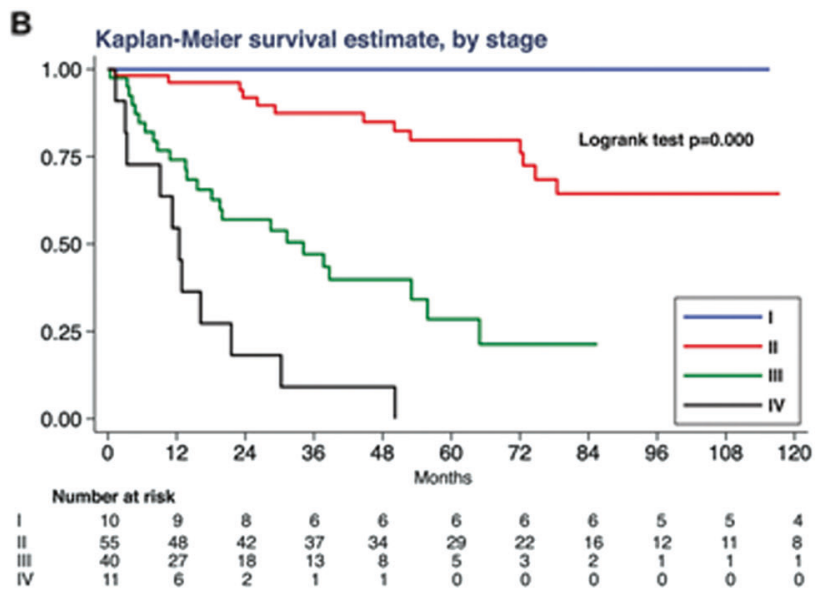

D

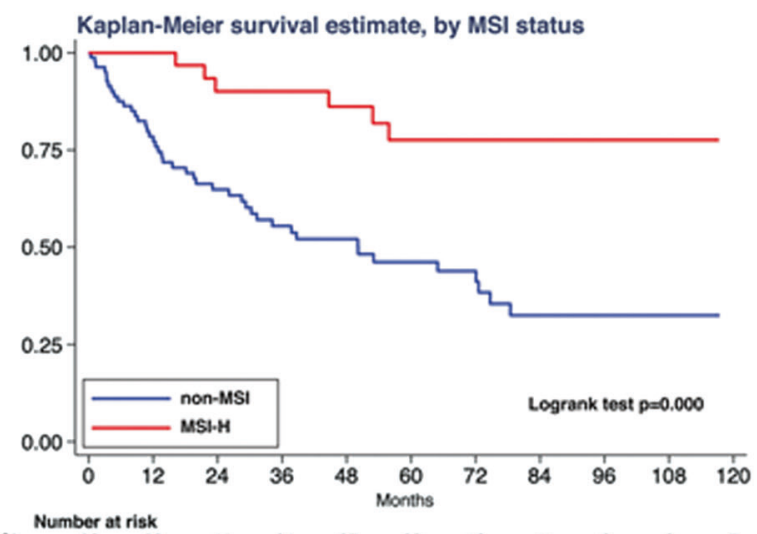
$\begin{array}{lccccccccccc}\text { non-MSI } & \text { Number at risk } & & & & & & & & & & \\ \text { MSI-H } & 36 & 33 & 44 & 34 & 27 & 23 & 16 & 11 & 9 & 9 & 7 \\ & 36 & 33 & 27 & 22 & 17 & 15 & 13 & 9 & 8 & 6\end{array}$

$\mathbf{F}$

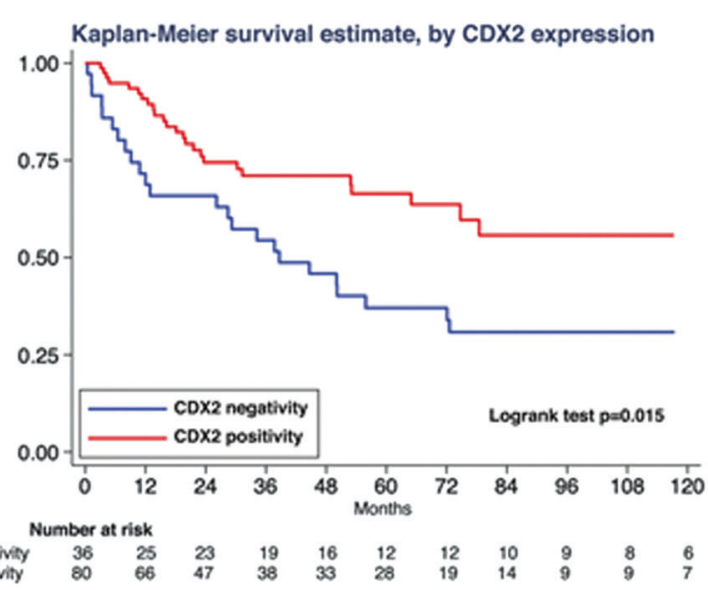

expression f). CeD-SBA, celiac disease-associated small bowel adenocarcinoma; CrD-SBA, Crohn's disease-associated small bowel adenocarcinoma.

PD- $1^{+}$immune cells observed in CeD-SBAs might be secondary to the underlying immune responses. In patients with untreated $\mathrm{CeD}$ without SBA, PD-L1 is reported to be overexpressed in enterocytes and lamina propria mononuclear cells, whereas PD-1 is not present in the small bowel [30]. In CrD patients without SBA, PD-L1 is decreased in $\mathrm{CD}^{+}$ 


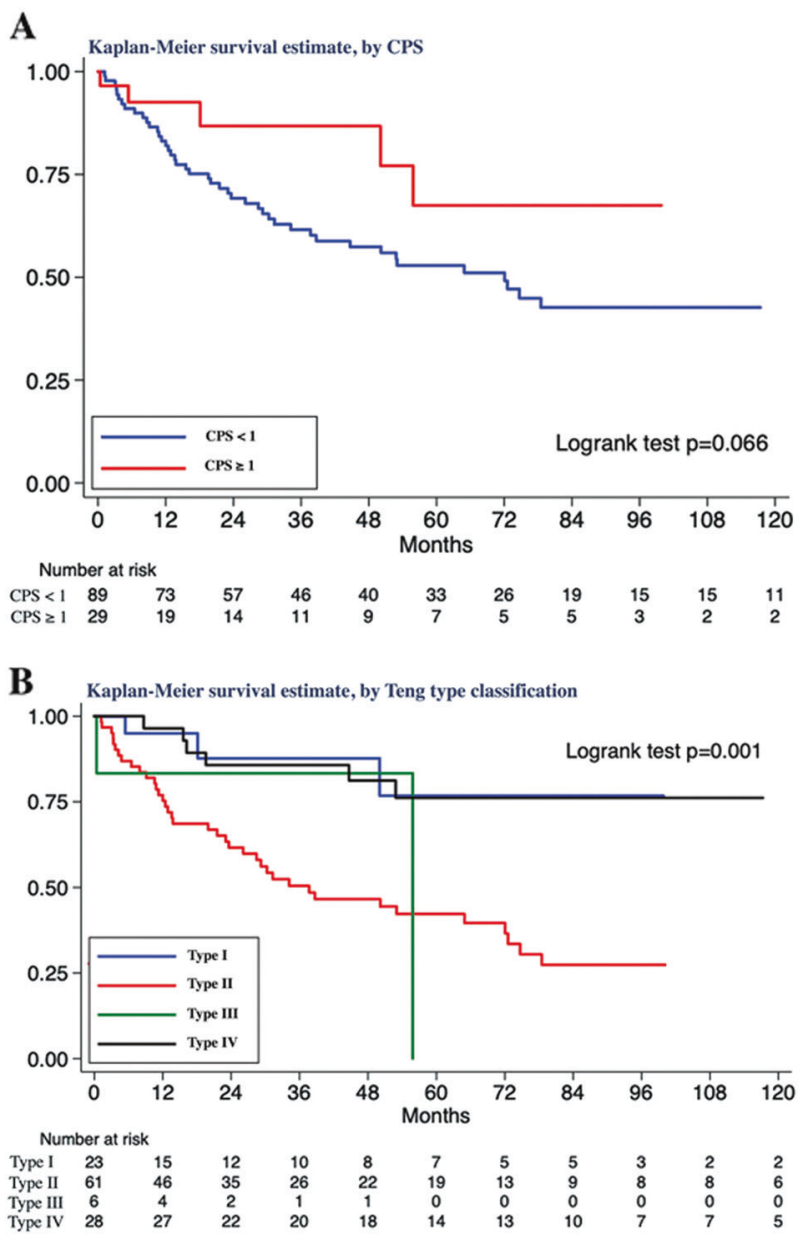

Fig. 3 Kaplan-Meier cancer-specific survival estimates for small bowel adenocarcinoma (SBA) patients. Survival estimates by PD-L1 expression according to combined positive score (CPS) a and Teng type classification $\mathbf{b}$.

$\mathrm{T}$ cells owing to high expression of the $\mathrm{T}$ helper 1 transcription factor T-bet via transfection [31], whereas another study [32] showed upregulation of PD-L1 in macrophages and PD-1 in $\mathrm{T}$ cells, mostly $\mathrm{CD} 4^{+}$, and $\mathrm{B}$ cells from the lamina propria. It must be outlined that the expression of PD-L1 and PD-1 we scored in our series was strictly related topographically (within tumor tissue or immediately surrounding it) to SBA, not to the underlying inflammatory $\mathrm{CeD}$ or $\mathrm{CrD}$ background. Whether the use of anti-PD-L1 or anti-PD-1 monoclonal antibodies in $\mathrm{CeD}$ and/or $\mathrm{CrD}$ patients induces changes of immunemediated intestinal disorder, which might also affect SBA, requires more-in-depth study [33].

As interferon- $\gamma$, a cytokine known to be implicated in the pathogenesis of both $\mathrm{CeD}$ and $\mathrm{CrD}[19,34]$, reduced in vitro expression of L-FABP from colorectal cancer cell lines [24], we investigated L-FABP in our cohort. In keeping with colorectal cancer [24], we demonstrated that L-FABP negativity is more frequent in MSI-H SBAs than in microsatellite stable cases. In addition, we found that PD-L1 ${ }^{+}$ SBAs correlated with L-FABP negativity. As in colorectal carcinomas, the lack of expression of the intestinal marker CDX2 is known to be associated with PD-L1 expression [16], we also correlated PD-L1 with CDX2 expression. However, we did not find a significant association between PD-L1 expression with CDX2 negativity.

We have also confirmed the previously reported association between MSI-H and increased expression of PD-L1 in SBAs [10-12], in keeping with findings in gastric and colorectal cancers [17, 35]. At first sight, this might shed light on MSI-H as a predictor of clinical response to immune checkpoint inhibitors in patients with SBA as well as in those with colorectal carcinoma [7, 8]. However, in agreement with endometrial carcinoma [14], we identified 15 PD-L1 ${ }^{+}$ microsatellite stable cases, i.e., $11 \mathrm{CrD}-\mathrm{SBAs}$, three $\mathrm{CeD}$ SBAs, and one sporadic SBAs, thus suggesting that MSI-H alone is not enough to detect all PD-L1 ${ }^{+}$SBAs. These findings strengthen the need to identify further predictors of response to immune checkpoint blockade. TILs have been recognized as a biomarker of host immune response against tumor [36], and their high density has been identified as an independent prognostic factor in non-hereditary SBAs [3, 12]. High TIL density, which is distinctive of both Teng types I and IV, contribute to adaptive resistance in cancers through the release of pro-inflammatory cytokines inducing PD-L1 expression and lastly triggering immunosuppression in the tumor microenvironment [6]. We here found that most PD-L1 ${ }^{+}$SBAs have an increased $\mathrm{CD}^{+}$and $\mathrm{CD} 8^{+} \mathrm{TIL}$ density. Notwithstanding, we identified seven out of 31 PD$\mathrm{L1}^{+}$cases with low TIL density, i.e., Teng type III. We speculate that tumor-specific molecular alterations might promote PD-L1 positivity in these latter SBAs.

PD-L1 expression is often coupled with EBV positivity in gastric cancer and two $\mathrm{EBV}^{+} \mathrm{CrD}-\mathrm{SBAs}$ have been reported [17, 25]. We here assessed latent phase EBV infection in our whole cohort and confirmed EBV positivity of the two previously reported cases while no EBV was detected in the remaining 116 cases tested. Among the two $\mathrm{EBV}^{+}$CrD-SBAs, the lymphoepithelioma-like carcinoma was $\mathrm{PD}-\mathrm{L1}^{+}$, whereas the glandular case was PD-L1negative. This is the first investigation showing no association between latent infection of EBV and CeD-SBA. In keeping with Von Rahden et al. [37], we confirmed EBV negativity in all sporadic SBAs. As regards histotypes, PD$\mathrm{L}^{+}$SBAs correlated with medullary histotype, known to have a favorable outcome not only in SBAs but also among gastric and colorectal cancers [38-40].

In our whole SBA series, PD-L1 expression according to CPS was associated with a trend toward a more favorable prognosis. This finding is not surprising, as PD-L1 expression was higher in SBAs with high $\mathrm{CD}^{+}$and $\mathrm{CD} 8^{+} \mathrm{TIL}$ density, and in those with medullary histotype, both parameters related to better prognosis [3, 40]. In addition, our findings confirmed etiologic group, histotype, TIL density, 
Teng tumor microenvironment immune types, and stage as prognostic factors in SBAs [2-4, 11, 12, 41]. Although PD$\mathrm{L}^{+}$cases according to a CPS showed a more favorable prognosis in comparison with $\mathrm{PD}-\mathrm{L}^{-}$patients at univariate analysis, PD-L1 expression lost its prognostic value when adjusted for TIL density. The main limitation of this study lies in its retrospective nature. However, owing to SBA rarity, this was a necessary choice to allow recruitment of a relatively wide cohort of SBAs.

In conclusion, PD-L1 expression in non-hereditary SBA is associated with its predisposing disease, being more frequent in CeD-SBA and CrD-SBA in comparison with sporadic SBA, as well as with its MSI status and TILs. The identification of a subset of PD-L1 ${ }^{+}$non-MSI cases in our cohort suggests that PD-L1 expression, along with MSI status, TIL density, and tumor mutation burden, should be considered as potential biomarkers of response to $\mathrm{PD}-1 / \mathrm{PD}-\mathrm{L} 1$ pathway blockade in well-designed clinical trials focused on SBA.

Acknowledgements This work was supported by the Fondazione IRCCS San Matteo Hospital-Italian Ministry of Health (Progetto di Ricerca Corrente 2019 to A.D.S.), the Fondazione IRCCS San Matteo Hospital (to O.L.) and the Italian Ministry of Education, University and Research (MIUR) (to the Department of Molecular Medicine of the University of Pavia under the initiative "Dipartimenti di Eccellenza (2018-2022)"). We thank Professor Francesco P. D'Armiento (Department of Advanced Biomedical Sciences, Federico II University of Naples, Naples, Italy), Professor Luigi Coppola (Unit of Pathologic Anatomy, San Filippo Neri Hospital, Rome, Italy), Dr. Antonio Maccioni (Pathology Unit, SS. Trinità Hospital, Cagliari, Italy) and Dr. Paola Migliora (Unit of Pathological Anatomy, Sant'Andrea Hospital, Vercelli, Italy) for providing us some formalin-fixed and paraffin-embedded samples and for taking part in the Small Bowel Cancer Italian Consortium. We thank Dr. Roberta Riboni (Anatomic Pathology Unit, Department of Molecular Medicine, University of Pavia and Fondazione IRCCS San Matteo Hospital, Pavia, Italy) for her support in molecular biology tests.

\section{Compliance with ethical standards}

Conflict of interest The authors declare that they have no conflict of interest.

Publisher's note Springer Nature remains neutral with regard to jurisdictional claims in published maps and institutional affiliations.

\section{References}

1. Halfdanarson TR, McWilliams RR, Donohue JH, Quevedo JF. A single-institution experience with 491 cases of small bowel adenocarcinoma. Am J Surg. 2010;199:797-803.

2. Giuffrida P, Vanoli A, Arpa G, Bonometti A, Luinetti O, Solcia E, et al. Small bowel carcinomas associated with immune-mediated intestinal disorders: the current knowledge. Cancers (Basel) 2018;11:1.

3. Vanoli A, Di Sabatino A, Furlan D, Klersy C, Grillo F, Fiocca R, et al. Small bowel carcinomas in celiac or crohn's disease: clinicopathological, molecular, and prognostic features. a study from the small bowel cancer italian consortium. J Crohns Colitis. 2017;11:942-53.
4. Vanoli A, Di Sabatino A, Martino M, Klersy C, Grillo F, Mescoli C, et al. Small bowel carcinomas in celiac or Crohn's disease: distinctive histophenotypic, molecular and histogenetic patterns. Mod Pathol 2017;30:1453-66.

5. Diosdado B, Buffart TE, Watkins R, Carvalho B, Ylstra B, Tijssen $\mathrm{M}$, et al. High-resolution array comparative genomic hybridization in sporadic and celiac disease-related small bowel adenocarcinomas. Clin Cancer Res. 2010;16:1391-401.

6. Sharpe AH, Pauken KE. The diverse functions of the PD1 inhibitory pathway. Nat Rev Immunol. 2018;18:153-67.

7. Chen C, Zhang F, Zhou N, Gu YM, Zhang YT, He YD, et al. Efficacy and safety of immune checkpoint inhibitors in advanced gastric or gastroesophageal junction cancer: a systematic review and meta-analysis. Oncoimmunology 2019;8:e1581547.

8. Le DT, Uram JN, Wang H, Bartlett BR, Kemberling H, Eyring $\mathrm{AD}$, et al. PD-1 blockade in tumors with mismatch-repair deficiency. N Engl J Med. 2015;372:2509-20.

9. Le DT, Durham JN, Smith KN, Wang H, Bartlett BR, Aulakh LK, et al. Mismatch repair deficiency predicts response of solid tumors to PD-1 blockade. Science 2017;357:409-13.

10. Thota R, Gonzalez RS, Berlin J, Cardin DB, Shi C. Could the PD1 pathway be a potential target for treating small intestinal adenocarcinoma? Am J Clin Pathol. 2017;148:208-14.

11. Watari J, Mitani S, Ito C, Tozawa K, Tomita T, Oshima T, et al. Molecular alterations and PD-L1 expression in non-ampullary duodenal adenocarcinoma: Associations among clinicopathological, immunophenotypic and molecular features. Sci Rep. 2019;9:10526.

12. Noh BJ, Hong SM, Jun SY, Eom DW. Prognostic implications of immune classification in a multicenter cohort of patients with small intestinal adenocarcinoma. Pathology 2020;52:228-35.

13. Salem ME, Puccini A, Grothey A, Raghavan D, Goldberg RM, Xiu J, et al. Landscape of tumor mutation load, mismatch repair deficiency, and PD-L1 expression in a large patient cohort of gastrointestinal cancers. Mol Cancer Res. 2018;16:805-12.

14. Crumley S, Kurnit K, Hudgens C, Fellman B, Tetzlaff MT, Broaddus R. Identification of a subset of microsatellite-stable endometrial carcinoma with high PD-L1 and CD8+ lymphocytes. Mod Pathol 2019;32:396-404.

15. Lee LH, Cavalcanti MS, Segal NH, Hechtman JF, Weiser MR, Smith JJ, et al. Patterns and prognostic relevance of PD-1 and PDL1 expression in colorectal carcinoma. Mod Pathol 2016;29:1433-42.

16. Inaguma S, Lasota J, Wang Z, Felisiak-Golabek A, Ikeda H, Miettinen M. Clinicopathologic profile, immunophenotype, and genotype of CD274 (PD-L1)-positive colorectal carcinomas. Mod Pathol 2017;30:278-85.

17. De Rosa S, Sahnane N, Tibiletti MG, Magnoli F, Vanoli A, Sessa $\mathrm{F}$, et al. $\mathrm{EBV}^{+}$and MSI gastric cancers harbor high PD-L1/PD-1 expression and high $\mathrm{CD}^{+}$intratumoral lymphocytes. Cancers (Basel). 2018;10:4.

18. Taube JM, Galon J, Sholl LM, Rodig SJ, Cottrell TR, Giraldo NA, et al. Implications of the tumor immune microenvironment for staging and therapeutics. Mod Pathol 2018;31:214-34.

19. Di Sabatino A, Corazza GR. Celiac disease. Lancet 2009;373:1480-93.

20. Gomollón F, Dignass A, Annese V, Tilg H, Van Assche G, Lindsay JO, et al. 3rd European Evidence-based consensus on the diagnosis and management of Crohn's disease 2016: Part 1: diagnosis and medical management. J Crohns Colitis. 2017;11:3-25.

21. Edge SB, Byrd DR, Compton CC, Fritz AG, Greene FL, Trotti A, Eds. AJCC cancer staging manual, 7th ed. New York, NY: Springer, 2010. p.127-32.

22. Chiaravalli AM, Feltri M, Bertolini V, Bagnoli E, Furlan D, Cerutti $\mathrm{R}$, et al. Intratumor $\mathrm{T}$ cells, their activation status and 
survival in gastric carcinomas characterized for microsatellite instability and Epstein-Barr virus infection. Virchows Arch 2006;448:344-53.

23. Kulangara K, Zhang N, Corigliano E, Guerrero L, Waldroup S, Jaiswal D, et al. Clinical utility of the combined positive score for programmed death ligand-1 expression and the approval of pembrolizumab for treatment of gastric cancer. Arch Pathol Lab Med. 2019;143:330-7.

24. Wood SM, Gill AJ, Brodsky AS, Lu S, Friedman K, Karashchuk $\mathrm{G}$, et al. Fatty acid-binding protein 1 is preferentially lost in microsatellite instable colorectal carcinomas and is immune modulated via the interferon $\gamma$ pathway. Mod Pathol 2017;30:123-33.

25. Vanoli A, Di Sabatino A, Martino M, Dallera E, Furlan D, Mescoli $\mathrm{C}$, et al. Epstein-Barr virus-positive ileal carcinomas associated with Crohn's disease. Virchows Arch 2017;471:549-52.

26. Teng MW, Ngiow SF, Ribas A, Smyth MJ. Classifying cancers based on T-cell infiltration and PD-L1. Cancer Res 2015;75:2139-45.

27. Arpa G, Grillo F, Giuffrida P, Nesi G, Klersy C, Mescoli C, et al. Separation of low versus high grade Crohn's disease-associated small bowel carcinomas is improved by invasive front prognostic marker analysis. J Crohns Colitis. 2019. [Epub ahead of print].

28. Rizzo F, Vanoli A, Sahnane N, Cerutti R, Trapani D, Rinaldi A, et al. Small-bowel carcinomas associated with celiac disease: transcriptomic profiling shows predominance of microsatellite instability-immune and mesenchymal subtypes. Virchows Arch. 2019. [Epub ahead of print].

29. Pedersen K, Foster N, Overman M, Boland P, Kim S, Arrambide K, et al. ZEBRA: an ACCRU/IRCI multicenter phase 2 study of pembrolizumab in patients with advanced small bowel adenocarcinoma (SBA). Ann Oncol. 2019;30. https://doi.org/10.1093/a nnonc/mdz154.006.

30. Ponce de León C, Angel López-Casado M, Lorite P, Palomeque $\mathrm{T}$, Isabel Torres M. Dysregulation of the PD-1/PD-L1 pathway contributes to the pathogenesis of celiac disease. Cell Mol Immunol. 2019;16:777-9.

31. Ma F, Zhao M, Song Z, Wang Z. T-bet interferes with PD-1/PDL1-mediated suppression of CD4(+) $\mathrm{T}$ cell inflammation and survival in Crohn's disease. Clin Exp Pharm Physiol. 2019;46:798-805.

32. Kanai T, Totsuka T, Uraushihara K, Makita S, Nakamura T, Koganei K, et al. Blockade of B7-H1 suppresses the development of chronic intestinal inflammation. J Immunol 2003;171:4156-63.

33. Cortellini A, Buti S, Santini D, Perrone F, Giusti R, Tiseo M, et al. Clinical outcomes of patients with advanced cancer and preexisting autoimmune diseases treated with anti-programmed death-1 immunotherapy: a real-world transverse study. Oncologist 2019;24:327-37.

34. Giuffrida P, Corazza GR, Di Sabatino A. Old and new lymphocyte players in inflammatory bowel disease. Dig Dis Sci. 2018;63:277-88.

35. Rosenbaum MW, Bledsoe JR, Morales-Oyarvide V, Huynh TG, Mino-Kenudson M. PD-L1 expression in colorectal cancer is associated with microsatellite instability, BRAF mutation, medullary morphology and cytotoxic tumor-infiltrating lymphocytes. Mod Pathol 2016;29:1104-12.

36. Mantovani A, Romero P, Palucka AK, Marincola FM. Tumor immunity: effector response to tumor and role of the microenvironment. Lancet 2008;371:771-83.

37. Von Rahden BH, Langner C, Brücher BL, Stein HJ, Sarbia M. No association of primary adenocarcinomas of the small bowel with Epstein-Barr virus infection. Mol Carcinog 2006;45:349-52.

38. Minamoto T, Mai M, Watanabe K, Ooi A, Kitamura T, Takahashi $\mathrm{Y}$, et al. Medullary carcinoma with lymphocytic infiltration of the stomach. Clinicopathologic study of 27 cases and immunohistochemical analysis of the subpopulations of infiltrating lymphocytes in the tumor. Cancer. 1990;66:945-52.

39. Friedman K, Brodsky AS, Lu S, Wood S, Gill AJ, Lombardo K, et al. Medullary carcinoma of the colon: a distinct morphology reveals a distinctive immunoregulatory microenvironment. Mod Pathol 2016;29:528-41.

40. Brcic I, Cathomas G, Vanoli A, Jilek K, Giuffrida P, Langner C. Medullary carcinoma of the small bowel. Histopathology 2016;69:136-40.

41. Jun SY, Park ES, Lee JJ, Chang HK, Jung ES, Oh YH, et al. Prognostic significance of stromal and intraepithelial tumorinfiltrating lymphocytes in small intestinal adenocarcinoma. Am J Clin Pathol 2020;153:105-18.

\section{Affiliations}

Paolo Giuffrida ${ }^{1}$ - Giovanni Arpa ${ }^{2}$. Federica Grillo ${ }^{3}$ Catherine Klersy ${ }^{4}{ }^{4} \cdot$ Gianluca Sampietro $^{5}$ - Sandro Ardizzone ${ }^{6}$. Paolo Fociani ${ }^{7}$ - Roberto Fiocca ${ }^{3} \cdot$ Giovanni Latella $^{8}$ - Fausto Sessa ${ }^{9}$. Antonietta D'Errico ${ }^{10}$ - Deborah Malvi ${ }^{10}$. Claudia Mescoli ${ }^{11}$ - Massimo Rugge ${ }^{11} \cdot$ Gabriella Nesi $^{12}$-Stefano Ferrero ${ }^{13}$ - Daniela Furlan $\mathbb{1}^{9} \cdot$ Gilberto Poggioli $^{14}$. Fernando Rizzello ${ }^{15}$. Maria C. Macciomei ${ }^{16}$. Donatella Santini ${ }^{10}$. Umberto Volta ${ }^{17} \cdot$ Roberto De Giorgio $^{18}$. Giacomo Caio ${ }^{18}$ - Antonio Calabrò ${ }^{19} \cdot$ Carolina Ciacci $\mathbb{1}^{20} \cdot$ Maria D'Armiento ${ }^{21} \cdot$ Aroldo Rizzo $^{22} \cdot$ Gaspare Solina $^{23}$. Michele Martino $^{1}$ - Francesco Tonelli ${ }^{24}$ - Vincenzo Villanaccii ${ }^{25}$ - Renato Cannizzaro ${ }^{26}$ - Vincenzo Canzonieri ${ }^{27,28}$. Ada M. Florena ${ }^{29} \cdot$ Livia Biancone ${ }^{30}$ - Giovanni Monteleone ${ }^{30}$. Roberto Caronna ${ }^{31}$ - Antonio Ciardi ${ }^{32}$. Luca Elli ${ }^{33}$. Flavio Caprioli ${ }^{33} \cdot$ Maurizio Vecchi $^{33} \cdot{\text { Renata } D^{\prime} \text { Incà }}^{34} \cdot$ Fabiana Zingone $^{34} \cdot$ Anna D'Odorico $^{34}$. Marco Vincenzo Lenti ${ }^{1}$ - Barbara Oreggia ${ }^{35}$ - Luca Reggiani Bonetti ${ }^{36}$ - Marco Astegiano ${ }^{37}$ - Elena Biletta ${ }^{38}$. Laura Cantoro ${ }^{39}$ - Antonino G. Giannone ${ }^{29}$ - Augusto Orlandi ${ }^{40}$. Claudio Papi ${ }^{41}$ - Vittorio Perfetti ${ }^{42}$. Erica Quaquarini ${ }^{43} \cdot$ Giancarlo Sandri $^{44} \cdot$ Marco Silano $^{45} \cdot$ Paolo Usai $^{46} \cdot$ Valeria Barresi $^{47} \cdot$ Rachele Ciccocioppo $^{48}$. Ombretta Luinetti ${ }^{1}$ - Paolo Pedrazzoli ${ }^{1,49}$. Andrea Pietrabissa ${ }^{50}$. Alessandra Viglio ${ }^{2}$. Marco Paulli ${ }^{2}$. Gino R. Corazza ${ }^{1}$ Enrico Solcia ${ }^{2} \cdot$ Alessandro Vanoli $^{2} \cdot$ Antonio Di Sabatino ${ }^{1}{ }^{1}$

1 Department of Internal Medicine, University of Pavia and Fondazione IRCCS San Matteo Hospital, Pavia, Italy
Anatomic Pathology Unit, Department of Molecular Medicine, University of Pavia and Fondazione IRCCS San Matteo Hospital, Pavia, Italy 
3 Pathology Unit, Department of Surgical and Diagnostic Sciences, University of Genoa and San Martino/IST University Hospital, Genoa, Italy

4 Biometry and Statistics Service, Fondazione IRCCS San Matteo Hospital, Pavia, Italy

5 Unit of IBD Surgery, Luigi Sacco University Hospital, Milan, Italy

6 Gastroenterology Unit, Luigi Sacco University Hospital, Milan, Italy

7 Pathology Unit, Luigi Sacco University Hospital, Milan, Italy

8 Gastroenterology Unit, Department of Life and Enviromental Sciences, University of L'Aquila, L'Aquila, Italy

9 Pathology Unit, Department of Medicine and Surgery, University of Insubria, Varese, Italy

10 Department of Experimental, Diagnostic and Specialty Medicine (DIMES), Institute of Oncology and Transplant Pathology, University of Bologna, St. Orsola-Malpighi Hospital, Bologna, Italy

11 Pathology Unit, Department of Medicine, University of Padua, Padua, Italy

12 Division of Pathological Anatomy, Department of Surgery and Translational Medicine, University of Florence, Florence, Italy

13 Division of Pathology, Fondazione IRCCS Ca' Granda Ospedale Maggiore Policlinico, Department of Biomedical, Surgical and Dental Sciences, University of Milan, Milan, Italy

14 Surgery of the Alimentary Tract, Department of Medical and Surgical Sciences, Sant'Orsola-Malpighi Hospital, University of Bologna, Bologna, Italy

15 Intestinal Chronic Bowel Disease Unit, Department of Medical and Surgical Sciences, Sant'Orsola-Malpighi Hospital, Alma Mater Studiorum University of Bologna, Bologna, Italy

16 Pathology Unit, San Camillo-Forlanini Hospital, Rome, Italy

17 Department of Medical and Surgical Sciences, University of Bologna, Bologna, Italy

18 Department of Medical Sciences, University of Ferrara, Ferrara, Italy

19 Department of Experimental and Clinical Biomedical Sciences, University of Florence, Florence, Italy

20 Department of Medicine and Surgery, University of Salerno, Salerno, Italy

21 Public Health Department, Federico II University of Naples, Naples, Italy

22 Units of Pathology, Cervello Hospital, Palermo, Italy

23 Units of General Surgery, Cervello Hospital, Palermo, Italy

24 Surgery and Translational Medicine, University of Florence, Florence, Italy

25 Institute of Pathology, Spedali Civili Hospital, Brescia, Italy

26 Department of Gastroenterology, Centro di Riferimento Oncologico (CRO) di Aviano IRCCS, Aviano, Italy
27 Pathology Unit, Centro di Riferimento Oncologico (CRO) di Aviano IRCCS, Aviano, Italy

28 Department of Medical, Surgical and Health Sciences, University of Trieste, Trieste, Italy

29 Pathologic Anatomy Unit, Department of Health Promotion, Mother and Child Care, Internal Medicine and Medical Specialties, University of Palermo, Palermo, Italy

30 Department of Systems Medicine, University of Tor Vergata, Rome, Italy

31 Department of Surgical Sciences, La Sapienza University, Rome, Italy

32 Department of Radiological, Oncological, Pathological Sciences, Umberto I Hospital, La Sapienza University, Rome, Italy

33 Gastroenterology and Endoscopy Unit, Fondazione IRCCS Ca' Granda Ospedale Maggiore Policlinico, Milan, Italy

34 Gastroenterology Section, Department of Surgery, Oncology and Gastroenterology, University of Padua, Padua, Italy

35 General Surgery Unit, Ca' Granda-Ospedale Maggiore Policlinico, Milan, Italy

36 Section of Pathology, Department of Diagnostic Medicine and Public Health, University of Modena and Reggio Emilia, Modena, Italy

37 General and Specialistic Surgery, Città della Salute e della Scienza-Molinette Hospital, Turin, Italy

38 Anatomic Pathology ASL Biella, Biella, Italy

39 Gastroenterology Unit, USL Umbria 1, Perugia, Italy

40 Department of Biopathology and Image Diagnostics, University of Tor Vergata, Rome, Italy

41 IBD Unit, San Filippo Neri Hospital, Rome, Italy

42 Internal Medicine Unit, S.S. Annunziata Hospital, ASST-Pavia, Varzi, Italy

43 Medical Oncology Unit, IRCCS ICS Maugeri and Department of Internal Medicine and Therapeutics, University of Pavia, Pavia, Italy

44 Clinical Nutrition Unit, Sant'Eugenio Hospital, Rome, Italy

45 Unit of Human Nutrition and Health, Istituto Superiore di Sanità, Rome, Italy

46 Department of Internal Medicine, University of Cagliari, Cagliari, Italy

47 Department of Diagnostics and Public Health, Section of Anatomical Pathology, University and Hospital Trust of Verona, Verona, Italy

48 Gastroenterology Unit, Department of Medicine, AOUI Policlinico G.B. Rossi, University of Verona, Verona, Italy

49 Oncology Unit, IRCCS San Matteo Hospital, Pavia, Italy

50 Department of Surgery, General Surgery II, University of Pavia and Fondazione IRCCS San Matteo Hospital, Pavia, Italy 